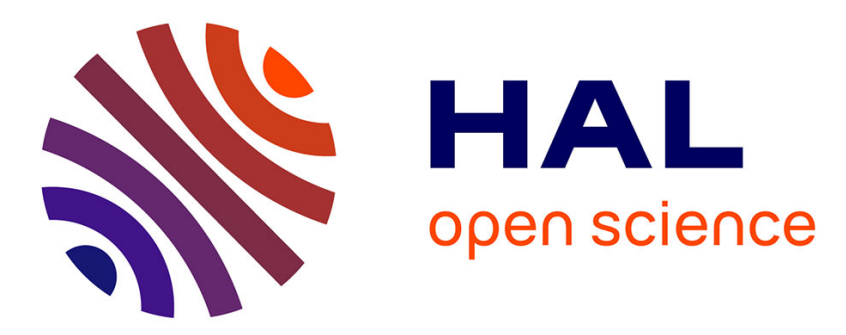

\title{
Kitting and time efficiency in manual assembly
}

Robin Hanson, Lars Medbo

\section{To cite this version:}

Robin Hanson, Lars Medbo. Kitting and time efficiency in manual assembly. International Journal of Production Research, 2011, 10.1080/00207543.2011.555786 . hal-00711440

\section{HAL Id: hal-00711440 \\ https://hal.science/hal-00711440}

Submitted on 25 Jun 2012

HAL is a multi-disciplinary open access archive for the deposit and dissemination of scientific research documents, whether they are published or not. The documents may come from teaching and research institutions in France or abroad, or from public or private research centers.
L'archive ouverte pluridisciplinaire HAL, est destinée au dépôt et à la diffusion de documents scientifiques de niveau recherche, publiés ou non, émanant des établissements d'enseignement et de recherche français ou étrangers, des laboratoires publics ou privés. 


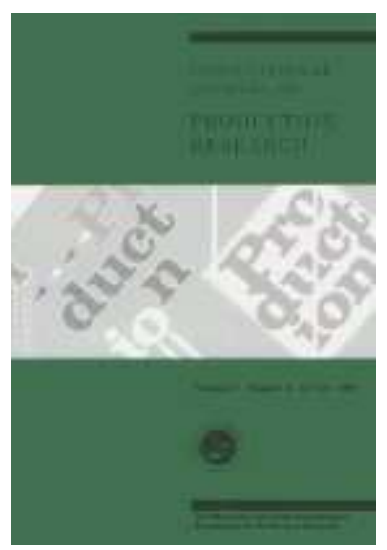

\section{Kitting and time efficiency in manual assembly}

\begin{tabular}{|r|l|}
\hline Journal: & International Journal of Production Research \\
\hline Manuscript ID: & TPRS-2010-IJPR-0802.R1 \\
\hline Manuscript Type: & Original Manuscript \\
\hline Date Submitted by the \\
Author: & 24-Nov-2010 \\
\hline $\begin{array}{r}\text { Complete List of Authors: } \\
\text { Keywords: }\end{array}$ & $\begin{array}{l}\text { Manson, Robin } \\
\text { Logistics and Transportation }\end{array}$ \\
\hline Keywords (user): & kitting, parts presentation \\
\hline
\end{tabular}

\section{SCHOLARONE} Manuscripts 


\title{
Kitting and time efficiency in manual assembly
}

\author{
Robin Hanson ${ }^{\mathrm{a}, \mathrm{b}^{*}}$ and Lars Medbo ${ }^{\mathrm{b}}$ \\ *Corresponding author \\ Robin Hanson (corresponding author) \\ R\&D Engineering, Saab Automobile \\ SE-461 80 Trollhättan, Sweden \\ Telephone: $+46(0) 733102270$ \\ Email: robin.hanson@saab.com \\ Lars Medbo \\ Division of Logistics and Transportation \\ Chalmers University of Technology \\ SE-412 96 Göteborg, Sweden \\ Telephone: $+46(0) 317721347$ \\ Email: lars.medbo@chalmers.se
}

${ }^{a} R \& D$ Engineering, Saab Automobile, Trollhättan, Sweden; ${ }^{b}$ Division of Logistics and Transportation, Chalmers University of Technology, Göteborg, Sweden

\begin{abstract}
This paper focuses on parts presentation in manual assembly. Its aim is to determine how kitting affects the time spent by the assembler fetching parts and, more specifically, what is the impact of the proportion of parts included in the kit. The paper is based on four case studies of automobile assembly, in which parts presentation by kitting is compared to parts presentation in component racks. In the case studies, kitting enabled shorter distances between parts presentation and assembly object and thereby a potential reduction in the time spent fetching parts. However, when only a proportion of parts were kitted, the time spent fetching parts was not always reduced, in spite of this potential. The paper finds that when deciding which parts should be kitted, attention should be paid to how and in what order assembly operations are performed.
\end{abstract}

Keywords: kitting; parts presentation; manual assembly 


\title{
Kitting and time efficiency in manual assembly
}

\begin{abstract}
This paper focuses on parts presentation in manual assembly. Its aim is to determine how kitting affects the time spent by the assembler fetching parts and, more specifically, what is the impact of the proportion of parts included in the kit. The paper is based on four case studies of automobile assembly, in which parts presentation by kitting is compared to parts presentation in component racks. In the case studies, kitting enabled shorter distances between parts presentation and assembly object and thereby a potential reduction in the time spent fetching parts. However, when only a proportion of parts were kitted, the time spent fetching parts was not always reduced, in spite of this potential. The paper finds that when deciding which parts should be kitted, attention should be paid to how and in what order assembly operations are performed.
\end{abstract}

Keywords: kitting; parts presentation; manual assembly

\section{Introduction and problem background}

In the context of in-plant materials supply, the principle of kitting is often discussed, as it has been stated to offer a number of advantages over the more traditional principle of continuous supply (also known as line-stocking). However, even though kitting has been used in industry for several years, relatively little research has examined its impact more specifically. The current paper focuses on the impact that kitting has on the work of the assembler in a manual assembly system and, more specifically, addresses the central aspect of time efficiency.

In contrast to continuous supply, where each part number is generally presented in a separate container, kitting means that parts are delivered and presented to the assembly operations in pre-sorted kits, with each kit containing parts for one assembly object. In many cases, each kit is delivered and presented in some form of carrier, such as a box or a trolley. The kit can be presented in a stationary position at an assembly station or, in case of assembly along an assembly line, the kit can travel with the assembly object along the line (Bozer and McGinnis 1992). Bozer and McGinnis (1992, p. 3) define a kit as "a specific collection of components and/or subassemblies that together (i.e. in the same container) support one or more assembly operations for a given product or 'shop order"'.

Compared to continuous supply, kitting has been reported to be associated with a number of potential advantages, such as space efficient parts presentation (Hua and Johnson 2010; Medbo 2003; Bozer and McGinnis 1992), improved assembly quality (Bozer and McGinnis 1992; Johansson 1991; Sellers and Nof 1986), shorter learning times (Johansson 1991) a more holistic understanding of the assembly work (Medbo 1999) and less time spent by the assembler fetching parts (Hua and Johnson 2010; Johansson 1991; Ding and Puvitharan 1990). Kitting is also associated with certain drawbacks. Most evident is the fact that the kits need to be prepared in advance, something that requires space and additional handling (Hua and Johnson 2010; Bozer and McGinnis 1992; Sellers and Nof 1986). Additional transportation may also be necessary if kits are prepared in a separate area not linked to either storage or assembly. 
Some aspects related to kitting have been studied, such as the allocation of parts to kits (Chen and Wilhelm 1997; Chen and Wilhelm 1993) and the efficiency of kit preparation and/or order picking operations (e.g. Christmansson et al. 2002; Brynzér and Johansson 1995; Tamaki and Nof 1991; Sellers and Nof 1986), but, as pointed out by Hua and Johnson (2010), there is a lack of knowledge about when and where kitting should be used. The possible effects of the use of kitting on the time efficiency of the assembly operations is one area that has not been thoroughly investigated. Furthermore, the fact that kitting can often be combined with continuous supply, meaning that an assembly station is supplied with some parts by kitting and others by continuous supply, is scarcely addressed in existing literature. An exception is Hua and Johnson (2010), who recognise the possibility of combining kitting and continuous supply, but who do not present any evidence as to the effects of such an approach.

One reason for combining kitting with continuous supply is to achieve the space savings at the assembly stations, associated with kitting, while at the same time not having to spend more time than necessary repacking parts into kits. With this rationale, it is mainly the parts that have many variants (and would thus require considerable space in component racks) that are included in the kits, while the parts with few variants can instead be supplied by continuous supply. However, it is likely to assume that the effects of using kitting are not the same when the kit contains all parts, compared to only a small proportion. Without knowledge of kitting and of the interplay between kitting and continuous supply, it is likely that applications in industry will not reach their full performance potential.

As seen in the background outlined above, there are a number of performance areas relevant in relation to kitting. In order to achieve depth in the analysis and results, this paper focuses on one performance area: time efficiency and, more specifically, the time spent by the assembler fetching parts. Accordingly, the aim of this paper is to determine how kitting affects the time spent by the assembler fetching parts and, more specifically, what is the impact of the proportion of parts included in the kit.

The paper is based on four case studies from two automobile assembly plants. Here, the use of kitting was studied and compared to the use of continuous supply. Each of the cases in the paper includes both a situation where all parts are presented at the assembly stations in component racks and another where some or all parts are instead presented in kits.

The remainder of the current paper is structured as follows: first, the frame of reference is presented, followed by the methodology used in the paper. Thereafter, the four cases are presented, followed by the findings in terms of the effects on fetching times when kitting is used in the different cases. The findings are then analysed and the results are presented. The paper concludes with a discussion of the study results and an outlook towards future research.

\section{Frame of reference}

In existing literature, a number of aspects of parts presentation have been reported as being relevant in relation to the time spent fetching parts in manual assembly. These are presented in the current section that functions as a frame of reference used in the paper.

In order to achieve efficient assembly operations in manual assembly, the valueadded share of the operators' work should be maximised. This is an issue that is often 
highlighted in the context of Lean Production (Bicheno 2004; Baudin 2002; Womack et al. 1990). The time spent fetching parts often constitutes a significant proportion of the non-value-added time spent by the assembler (Wänström and Medbo 2009) and should consequently be kept as short as possible, in order for efficiency to be achieved. Several aspects of the parts presentation are relevant in relation to the time spent fetching parts: the distance the assembler has to walk in order to reach the part (Wänström and Medbo 2009; Finnsgård et al. 2008), the type of unit load used for presenting the parts (Hanson 2011; Finnsgård and Wänström 2009), the height and orientation of the unit loads (Finnsgård and Wänström 2009) and how the parts are grouped, e.g. whether they are sorted in kits or according to part number (Hua and Johnson 2010, Johansson 1991). According to Baudin (2002, p. 64), parts "should be presented to assemblers unpacked, within arm's reach, with their smallest dimensions facing out, and oriented for easy installation". This way, the assembly operations are facilitated and non-value-added time can be reduced.

Presentation of parts by kitting, compared to by continuous supply, seems to hold a potential for reducing the time spent by the assembler fetching parts. Two separate contributing factors can be identified: 1) kitting eliminates the time needed for searching for parts (Hua and Johnson 2010; Johansson 1991; Ding and Puvitharan 1990) and 2) kitting often enables the parts being presented in a suitable picking position relative to the assembly object (Deechongkit and Srinon 2009; Neumann et al. 2006; Jonsson et al. 2004).

\section{Methodology}

As previously stated, literature dealing with the specific effects of presenting parts by kitting, compared to by continuous supply, is scarce. Addressing the existing knowledge gaps regarding how kitting affects the time spent fetching parts in manual assembly, this paper presents four case studies from two companies within the automotive assembly industry, where kitting has been used. The current section presents the four cases and explains their relevance in relation to the aim of the paper. Furthermore, the section presents the methods used in the data collection and analysis of these cases.

\subsection{Case selection}

In line with the aim of the paper, focusing on determining how kitting affects the time spent by the assembler fetching parts, four cases were identified and selected where the use of kitting could be compared to the use of continuous supply. In one of the cases, in the paper denoted case 1, a transition was made from kitting to continuous supply, whereas in each of the other three cases, in the paper denoted case 2 , case $3 a$ and case $3 b$, a transition was made from continuous supply to kitting. In all of the four cases, it was possible to study assembly operations in more or less the same settings, but with different materials feeding principles. This resulted in an excellent basis for comparison between the effects of using kitting and continuous supply on the time spent fetching parts. As the paper further seeks to identify the impact of the proportion of parts included in the kit, the cases studied were also chosen so that they among themselves displayed differences in this respect. Accordingly, the kits used in case 1 included $100 \%$ of the parts, the kits in case 2 included $50 \%$ of the parts, the kits in case $3 \mathrm{a}$ included $22 \%$ of the parts, and the 
kits in case $3 \mathrm{~b}$ included $10 \%$ of the parts. The parts that were not presented in kits were instead presented in component racks.

\subsection{Methods for data collection and analysis}

In all four cases, the work of the assemblers was video-recorded and analysed. An approach was used similar to that of Engström and Medbo (1997), in which manual assembly work is recorded in detail and analysed using a computer synchronised with the video recorder. With this approach, the output of the initial analysis of the video recordings is a categorisation of the recorded work into predefined activities, in which the time consumption of each activity is registered. In the current paper, the activities the work was divided into were the same as the ones used by Jonsson et al. (2004) and accordingly included the activities direct assembly work, fetch small materials, fetch medium sized materials, and fetch large materials. In the current paper, the time spent fetching medium-sized and large materials constitutes the basis for the figures presented and analysed. Included in fetching are all activities performed by the assembler in association with getting a part from where it is presented to where it is to be assembled, i.e. turning, walking, reaching out, grasping and walking back to the assembly object.

Not all activities performed by the assemblers are included in the analyses, as some are not relevant to the aim of the paper. Notable is the exclusion of the activity "fetch small materials", included in the original analysis of the video recordings. The picking of small materials (a term that mainly denotes fasteners, such as screws and bolts) differed to the picking of the other, larger components. In all of the cases, small materials were supplied by continuous supply, both in the kitting and non-kitting situations. Furthermore, unlike the larger components, the small materials were often picked several at a time (i.e. in one movement of the assembler). In the video analyses it was therefore difficult to determine how many parts were actually picked in these activities. Accordingly, in the presentation of the case studies in this paper, where the percentage of parts included in the kits is listed, this figure does not consider small materials.

As each of the case studies includes two different situations, one where kitting was used and one where it was not, the assembly work was recorded at two occasions for each case. Each recording was performed during a workday chosen at random. Table 1 shows the number of assembly cycles recorded for each of the case studies. In line with the purpose of the paper, the analysis is based on the average fetching times recorded in each of the cases. In order to enable an understanding of the origin of these fetching times, the analysis further considers the number of parts fetched from both component racks and kits, as well as the number of times the assembler visited the component racks, as this was closely associated with the walking distances of the assembler.

In two of the cases studied, cases $3 a$ and $3 b$, the length of the assembly stations was reduced at the same time that kitting was introduced. (This was not related only to the introduction of kitting, but also to a simultaneous reduction in assembly cycle time. However, the introduction of kitting helped enabling the reduction of the length of the assembly stations, as the component racks were reduced when parts were presented in kits instead of in component racks.) In both of these cases, assembly was performed along a continuously moving assembly line. By the company, the reduction in assembly station length was considered to impact the work of the assemblers mainly by decreasing the walking distance preceding each work cycle (when the assembler returns to the start 
of the assembly station, after having followed the preceding assembly object to the end of the assembly station). The time spent by the assemblers walking back to the start of the assembly station at the beginning of each assembly cycle was not included in the analyses of the current paper, focusing only on the time spent fetching parts, and the potential effects of the change in assembly station length should therefore be negligible in relation to the aim of the paper.

Table 1. Number of assembly cycles recorded and analysed in each case study.

It is sometimes argued that the external validity and reliability of case studies are limited, affecting the generalisability of the results. However, as pointed out by Yin (2003), case studies rely on analytical generalisation as opposed to statistical generalisation. In the current paper, the descriptions provided of each case make it possible to gain an understanding of what factors lie behind the observed effects, which in turn makes it possible to apply the results from the case studies to other situations.

\section{Presentation of the cases}

As previously stated, all four cases presented in this paper are from the automobile assembly industry. Assembly was in all cases manual and performed according to masscustomisation principles, where products were assembled in mixed sequence in terms of model, variant and colour. Accordingly, for several of the assembled parts there were several variants, which differed from product to product.

Cases 2, 3a and $3 \mathrm{~b}$ are all from the same company. In these three cases, kitting was introduced at the same time as the respective assembly lines were rebalanced as part of an increase in production pace for the production plant as a whole. The increase in assembly pace and the introduction of kitting were initiated for different reasons and were not directly related. However, both the increase in assembly pace and the introduction of kitting were by the company found to require rebalancing of the assembly line. The reason that the introduction of kitting was found to require rebalancing of the assembly line was that the walking distances and the time for fetching parts were expected to be reduced. To avoid having to rebalance the assembly line twice, the decision was made to introduce the two changes at the same time. As a result of the rebalancing of the assembly line, there were changes in how many and what parts were assembled at each station. In each of the case presentations below, information showing these changes is presented, making it possible to discern which changes in performance were actually the result of kitting.

\subsection{Case 1}

Case 1 comprises the complete assembly of the front and rear doors of a car. The case study is based on both an initial situation where assembly was performed in stationary cells, with parts supplied and presented in stationary kits, and a later situation, where a transition had been made to assembly along a continuously moving assembly line, with parts presented in component racks along the assembly line. Before the transition, the cycle time for assembling one pair of doors was 13 minutes. After the transition to 
assembly along an assembly line, the basic cycle time was 1.0 minute. Some assembly operations were, however, performed in longer cycles, corresponding to two, three or four times the basic cycle time; i.e. the assemblers followed the assembly objects over distances that were two, three or four times the distance corresponding to the basic cycle time.

In the initial situation using kitting, parts were presented to the assembler on small trolleys placed within arm's length of the assembly object. Each trolley had multiple shelves and compartments and contained parts for two pairs of doors. Not included on these trolleys were small materials such as nuts and bolts, which were instead supplied by continuous supply and presented in component racks. In this case these were also within arm's length of the assembly object. As stated in the methodology section, small materials are not included in the analysis of any of the cases studied, however.

After the transition to assembly along an assembly line, all parts were presented in component racks, meaning that the proportion of kitted parts was reduced from $100 \%$ to $0 \%$. Assembly was performed on two different assembly lines, one for the right-hand side and one for the left-hand side of the car. The doors travelled along the assembly lines in pairs (front and rear for the same side of the car), hanging on a carrier from a conveyor. There were 15 assembly stations on each assembly line. The component racks were placed along the assembly line, on the opposite side of the assembler relative to the assembly object, requiring the assembler to turn and walk to fetch parts. The distance between an assembly object and the component racks was 1.7 metres. The parts were presented in different types of packaging within the component racks. Some were presented in plastic boxes, others in large pallets with collars, and yet others were delivered in production sequence and presented in component racks designed for these parts. Table 2 presents an overview of the characteristics of case 1 .

In case 1 , when assembly was performed along a continuously moving assembly line and parts were presented in component racks, it was observed that the assemblers were frequently ahead of schedule, meaning that they were performing assembly operations before they were supposed to, according to how production was planned and how the assembly line was balanced. As is generally the case in manual assembly along a continuously moving assembly line, the parts presentation in the component racks was arranged so that the parts needed early in each assembly cycle were presented at the beginning of the respective assembly station, whereas parts needed later in the assembly cycle were instead presented further down the assembly station. This meant that the distance for fetching parts was as short as possible if the assembler performed the assembly operations in each assembly cycle at exactly the planned pace. Consequently, if the assemblers, as in this case, worked ahead of schedule, the time for fetching parts would increase, compared to plan.

Table 2. Overview of the characteristics of case 1. 


\subsection{Case 2}

Case 2 comprises the operations in one assembly station on a continuously moving assembly line, where instrument panels were assembled. The introduction of kitting coincided with a reduction in cycle time from 2.7 to 2.0 minutes and a rebalancing of the assembly line. As a result of the reduced cycle time, the number of assembly stations on the assembly line was increased from 10 to 13 . Consequently, there was a change in how many and, to some extent, what assembly operations were performed at each assembly station and, in line with this, the assembly of many parts was moved between stations.

Before the introduction of kitting, all parts were presented in component racks to which the assembler needed to turn and walk in order to fetch parts. The distance between assembly object and component racks was 1.1 metres. The introduced kit was contained in a plastic box that was hung on the carrier of the instrument panel and accordingly travelled with the assembly object along the assembly line, containing parts for several assembly stations. The kit was placed immediately next to, and within arm's length of, the instrument panel.

Before the introduction of kitting and rebalancing of the assembly line, nine parts were assembled at the station. After the introduction of kitting and the associated rebalancing of the assembly line, only six parts were assembled. Four of these parts were the same before and after the introduction. After the introduction of kitting, three parts were included in the kit and three were supplied by continuous supply. This means that the proportion of parts included in the kit was 50\%. All three of the parts included in the kit were assembled at the station already before the introduction of kitting. An overview of the characteristics of case 2 is presented in table 3.

Table 3. Overview of the characteristics of case 2.

\subsection{Cases $3 a$ and $3 b$}

Cases $3 \mathrm{a}$ and $3 \mathrm{~b}$ are from the same assembly line but each comprises the operations in a separate assembly station. The assembly was performed along a continuously moving assembly line, where front and rear doors were assembled. The front and rear doors of the right and the left-hand side of the car were assembled on either side of the assembly line. The doors travelled along the assembly line in pairs (front and rear for the same side of the car), hanging on a carrier from a conveyor. Kitting was introduced at the same time as a reduction in cycle time from 2.1 to 1.6 minutes, at which point the assembly line was also rebalanced. In association with the rebalancing of the assembly line, the number of assembly stations on either side of the line was increased from 13 to 18 . Consequently, just like in case 2 , there was a change in how many and what assembly operations were performed at each assembly station and, in line with this, the assembly of many parts was moved between stations.

Before the introduction of kitting, all parts were presented in plastic boxes in component racks along the assembly line. To fetch parts, the assembler needed to turn and walk to the component racks. The distance between a pair of doors and the component racks was 1.1 metres. The introduced kit was contained in a plastic box hung 
between the front and rear doors on the same carrier, meaning that the kit parts were within arm's length of the doors. The kit travelled with the assembly object along the assembly line and contained parts for several assembly stations.

\subsubsection{Case $3 a$}

At the assembly station in focus here, a number of operations were performed on the right-hand rear door. As a result of the introduction of kitting and the associated rebalancing of the assembly line, one of the parts previously assembled at the station was moved to another station, meaning that only nine of the original ten parts remained. Of these nine parts, two were included in the new kit. The proportion of parts kitted at the station was accordingly $22 \%$. Table 4 presents an overview of the characteristics of case $3 a$.

Table 4. Overview of the characteristics of case $3 \mathrm{a}$.

\subsubsection{Case $3 b$}

Case $3 \mathrm{~b}$ focuses on another assembly station on the same door assembly line as case $3 \mathrm{a}$ above. Here, however, assembly focused on the right front door. Before the introduction of kitting and rebalancing of the assembly line, seven parts were assembled at the station in each assembly cycle. After the introduction of kitting, ten parts were assembled at the station, three of which were identical to those before the changes. One of these three was included in the kit, meaning that the proportion of parts included in the kit was $10 \%$. Table 5 presents an overview of the characteristics of case $3 \mathrm{~b}$.

Table 5. Overview of the characteristics of case $3 b$.

\section{Observed effects of using kitting}

In each of the cases, the time spent fetching parts was measured and registered according to the approach described in the methodology section, i.e. based on the video recordings of the work performed at the assembly stations. In each of the cases, the total time spent by the assembler fetching parts was registered and then divided by the number of parts fetched, resulting in an average time spent fetching each part. Accordingly, table 6 presents the effects identified in the case studies in terms of average fetching times when kitting was used and when it was not. For the situations when kitting was used, since kitting was combined with continuous supply in cases 2 , $3 \mathrm{a}$ and $3 \mathrm{~b}$, the tables also include separate figures for the average fetching times from kits and from component racks. As the assembler in each of the cases sometimes fetched more than one part when visiting the component racks, the average number of visits to the component racks each assembly cycle is presented for each of the cases, as is the average number of parts fetched each assembly cycle. The number of visits to the component racks was closely 
related to the walking distance of the assembler and is therefore highly relevant in relation to the time spent fetching parts. As the kit in each of the cases was presented within arm's length of the assembly object, practically no walking was required for fetching parts from kits.

Table 6. Overview of the findings of the four case studies - fetching times.

\section{Analysis}

The current section presents an analysis of the effect of using kitting that were observed in the cases and presented in the previous section. First, each case is analysed separately and, thereafter, a cross-case analysis is presented, where similarities and differences between the identified effects in four cases are related to the characteristics of the cases.

\subsection{Within-case analysis}

\subsubsection{Case 1}

In the first situation, when assembly was performed in stationary cells and where all parts were presented in kits, the average time spent by the assembler fetching each part was $63 \%$ lower than for the later situation using assembly along a continuously moving line, with all parts presented in component racks $(2.81 \mathrm{~s}$ for kits, compared to $7.55 \mathrm{~s}$ for component racks). This reduction in fetching times is in line with expectations. As stated in section 1, the time spent searching for parts is shorter when kitting is used compared to continuous supply. Furthermore, in this case, kitting was associated with a shorter distance between the assembly object and the position where the parts were presented. As further noted in this case, the walking distances for fetching parts were often prolonged in the situation when parts were presented in component racks, as the assemblers were often ahead of schedule and thus fetched parts before the assembly object (that moved continuously) had reached its optimal position along the component racks. In contrast, when kitting was used, both the kits and the assembly objects were stationary at what had been decided to be the most favourable distance.

\subsubsection{Case 2}

After the simultaneous introduction of kitting and rebalancing of the assembly line, the average fetching time per part was reduced by $43 \%$ (1.86 s compared to $3.29 \mathrm{~s}$ ). The average time it took to fetch a part from the kit was 56\% lower than the average time to fetch a part from the component racks before the introduction of kitting (1.46 s for kit, compared to $3.29 \mathrm{~s}$ for component racks). The average time required to fetch a part from the component racks at the studied assembly station was reduced by $31 \%$ after the introduction of kitting and rebalancing (2.26 s after, compared to $3.29 \mathrm{~s}$ before).

As in case 1, the reduction in average time spent fetching parts from kits compared to from component racks is in line with expectations. More surprising is the reduction in the average time spent each part from the component racks. However, before the introduction of kitting, the assembler visited the component racks seven times per assembly cycle to 
fetch the nine parts for assembly. After the changes, the assembler needed to visit the component racks only once per assembly cycle to fetch the three parts presented there, which meant that the average number of parts fetched on each visit to the component racks increased. Naturally, in terms of average fetching times, it is advantageous for the assembler if it is possible to pick several parts on each visit to the component racks. Whether or not this is possible depends, as stated by production engineers within the case company, on the physical characteristics of the parts as well as on the sequence in which they are assembled; if it is not possible to temporarily place parts on or by the assembly object, it is necessary that all parts fetched on each visit to the component racks are assembled right after each other and that it is possible for the assembler to carry them all whilst performing assembly. Accordingly, the reduced time for fetching parts from the component racks was not a direct result of the parts presentation in terms of materials feeding principle, but rather of how well the parts presentation matched the assembly operations and the order in which the different parts were fetched.

\subsubsection{Case $3 a$}

After the simultaneous introduction of kitting and rebalancing of the assembly line, the average fetching time per part was increased by $2 \%$ (3.14 s after, compared to $3.07 \mathrm{~s}$ before). The average time it took to fetch a part from the kit was $63 \%$ lower than the average time to fetch a part from the component racks before the introduction of kitting (1.14 $\mathrm{s}$ for kit, compared to $3.07 \mathrm{~s}$ for component racks). The average time required to fetch a part from the component racks was instead increased by $31 \%$ (3.71 s after the introduction of kitting, compared to $3.07 \mathrm{~s}$ before).

As can be seen from the figures, the time required for fetching the kitted part was reduced as expected, but for the parts that were presented in component racks, the average fetching times were instead increased. Both before and after the introduction of kitting and the rebalancing of the assembly line, the assembler visited the component racks three times per assembly cycle, but the number of parts fetched from these racks had decreased from ten to seven, i.e. the average number of parts fetched on each visit to the component racks had decreased.

\subsubsection{Case $3 b$}

After the simultaneous introduction of kitting and rebalancing of the assembly line, the average time spent by the assembler fetching each part was reduced by $7 \%(2.19 \mathrm{~s}$ after, compared to $2.35 \mathrm{~s}$ before). For parts included in the kit, the average fetching time was $58 \%$ shorter than for those fetched from component racks before the introduction of kitting ( $0.99 \mathrm{~s}$ for parts from kit, compared to $2.35 \mathrm{~s}$ for parts from component racks). The average time for fetching each of the parts presented in the component racks was reduced by $1 \%$ ( $2.32 \mathrm{~s}$, compared to $2.35 \mathrm{~s}$ ) in the case study, something that cannot be attributed directly to kitting. It can be noted that the assembler visited the component racks to fetch parts four times each assembly cycle both before and after the introduction of kitting, but the number of parts fetched increased from seven to nine per cycle when kitting was introduced.

\subsection{Cross-case analysis}


In all four cases, the parts presented in kits were better positioned relative to the assembly object than were the parts presented in component racks; the kits were presented both at a shorter distance from the assembly object and in a position to which the assembler did not need to turn in order to reach. It is therefore natural that, in all four cases, the average fetching time was much shorter for a part presented in a kit compared to one presented in a component rack. It is, however, difficult to determine whether the shorter time for fetching parts was a result only of the better picking position. As stated in section 2, kitting also means that no time needs to be spent by the assembler searching for parts (Hua and Johnson 2010; Johansson 1991; Ding and Puvitharan 1990).

As previously stated, the current paper pays particular interest to the interplay between kitting and continuous supply when these two materials feeding principles are combined at an assembly station. As the average fetching time per part in the cases was shorter for parts presented in kits compared to parts presented in component racks, a simple assumption could perhaps be that the higher the proportion of parts kitted, the shorter the average fetching time per part. As revealed by the case studies, however, this was not true. Instead, the average fetching time per part differed substantially between the four cases, with no direct relation to the proportion of parts kitted. This resulted mainly from large variations in the average fetching times between the different parts presented in component racks. These variations tended to overshadow the fact that the average fetching times from kits were relatively short in all four cases. In case 1, where a comparison was made between having all parts presented in kits and, instead, having all parts presented in component racks, the advantages of having all parts presented close to the assembly object were evident.

Considering only the parts presented in component racks, a feasible explanation to the varying average fetching times is the average distance that the assembler needs to cross in order to fetch each part; something that, in turn, is related to the number of parts that the assembler fetches on each visit to the component racks. Since an assembler often fetches more than one part per visit to the component racks, it is not certain that kitting one additional part will reduce the number of visits to the component racks by one. This was evident in case study 3a, where the introduction of kitting was associated with changes in average fetching times that could not be anticipated simply on the basis of the proportion of parts kitted. Here, the average fetching time per part was actually slightly longer when $22 \%$ of the parts were kitted than when all parts were presented in the component racks. The reason for this was that even though the number of parts fetched from component racks was reduced when kitting was introduced, the assembler still needed to visit the component racks the same number of times per assembly cycle.

\section{Results}

In the cases studied, the fetching times were considerably shorter for parts presented in kits compared to parts presented in component racks. It is clear that the time spent fetching parts is greatly affected by the walking distance of the assembler, which, in turn, is closely related to the number of visits to the component racks. The case studies have further shown that when kitting is combined with continuous supply, the number of visits to the component racks is not directly linked to the proportion of parts included in the kits, which indicates a need to consider how the assembler operations are performed in order to achieve the greatest benefit in terms of time spent fetching parts. 


\section{Discussion and issues for future research}

The current paper has provided information regarding the effects of using kitting to supply and present parts in manual assembly. This information is important, since there is currently a lack of knowledge regarding when and where kitting should be used. More specifically, hardly any previous literature addresses the combination of kitting and continuous supply, which is in focus in the current paper. Based on the case studies, it is clear that, when using kitting in combination with continuous supply, it is necessary to consider the relation between these two materials feeding principles in order to determine a satisfactory overall solution. From the perspective of the time consumption of the assembler, each part that is presented in a kit, rather than in a component rack further from the assembly object, should be associated with a reduction in the number of visits to the component racks.

It should be noted that in addition to distance between parts presentation and assembly object, and the number of visits to the component racks, a number of further aspects exist that could impact the time spent by the assembler fetching parts. As stated in existing literature (Hua and Johnson 2010; Johansson 1991; Ding and Puvitharan 1990), one of the advantages of kitting is that less time needs to be spent searching for parts. Furthermore, it is important to recognise that there can be differences in how the parts are presented also within the options of kitting and continuous supply, i.e. the fetching times can differ between different configurations of continuous supply or between different configurations of kitting. This is evident when comparing the fetching times in case 1 to the fetching times in the other cases. The fetching times are considerably longer in case 1 compared to the other cases - both when parts are presented in kits and in component racks. In the situation when all parts were presented in component racks, it was observed that the assemblers were frequently ahead of schedule and, as a result, that the walking distances for fetching parts were longer. Furthermore, the distance between the assembly object and the component racks was longer in case 1 than in the other cases. Naturally, these aspects contributed to the fetching times from component racks being much longer in case 1 than in the other cases. Similarly, in the situation when all parts were presented in kits, each kit was in case 1 presented in a trolley. In the other three case studies, where a smaller proportion of the parts are kitted, the kits were instead presented in boxes. The difference in time saved could suggest that kits containing a large number of parts might be difficult to present in a suitable picking position and that the design of such kits deserves particular attention.

A feature common to all the cases examined in this paper was that the parts presented in kits were placed much closer to the assembler than those presented in component racks. It should be noted that kitting applications exist where the kits are presented on the opposite side of the assembler relative to the assembly object. In such contexts, the parts might not be presented any closer to the assembly object than they would have been in a component rack.

As stated at the beginning of this paper, kitting is associated with a number of potential effects. Most of these have not been dealt with in the case studies in this paper, which focuses only on the time spent by the assembler fetching parts. In order to make an informed decision of whether to use kitting or continuous supply in an industrial application, further knowledge is needed of performance in terms of space efficiency, inventory levels, assembly quality and learning times. Hence, further research is required, 
comparing the relative performance of kitting and continuous supply in these performance areas. Furthermore, it should be studied how combinations of kitting and continuous supply affect variables other than fetching time. Some of the potential benefits of kitting may be lost if a combinatory approach such as this is used. Beneficial learning effects and cognitive effects of kitting, based on the creation of a holistic understanding of the assembly object, may be difficult to achieve if only a proportion of the parts are kitted. In contrast, effects related to flexibility for product volume and mix, as well as for new product introductions and product modifications may probably be achieved, at least to some extent, when kitting is combined with continuous supply.

Furthermore, the effect of parts presentation on the time spent by the assembler fetching parts constitutes only one part of the total time-related effects of kitting. To gain a full picture, knowledge is needed of the time spent preparing the parts presentation, both for kitting and other materials feeding principles, and of how this time can be minimised. This is something that should be addressed in future studies. 


\section{References}

Baudin, M., 2002. Lean Assembly: The Nuts and Bolts of Making Assembly Operations Flow. New York: Productivity Press.

Bicheno, J., 2004. The new lean toolbox: towards fast, flexible flow, 3rd ed. Buckingham: PICSIE Books.

Bozer, Y.A. and McGinnis, L.F., 1992. Kitting versus line stocking: A conceptual framework and a descriptive model. International Journal of Production Economics, 28, 1-19.

Brynzér, H. and Johansson, M.I., 1995. Design and performance of kitting and order picking systems. International Journal of Production Economics, 41, 115-125.

Chen, J.F. and Wilhelm, W.E., 1993. An evaluation of heuristics for allocating components to kits in small lot, multi-echelon assembly systems. International Journal of Production Research, 31 (12), 2835-2856.

Chen, J.F. and Wilhelm, W.E., 1997. Kitting in multi-echelon, multi-product assembly systems with parts substitutable. International Journal of Production Research, 35 (10), 2871-2897.

Christmansson, M., Medbo, L., Hansson, G.A, Ohlsson, K., Bystrom, J.U., Moller, T. and Forsman, M., 2002. A case study of a principally new way of materials kitting an evaluation of time consumption and physical workload. International Journal of Industrial Ergonomics, 30 (1), 49-65.

Deechongkit, S. and Srinon, R., 2009. Three alternatives of material supply in assembly line: A comparative study. In: Proceedings of the 10th Asia Pacific Industrial Engineering and Management Systems Conference. Kitakyushu, Japan, 14-16 December 2009, 2062-2069.

Ding, F.-Y. and Puvitharan, B. (1990). "Kitting in just-in-time production", Production and Inventory Management Journal, 31(4), 25-28.

Engström, T. and Medbo, P., 1997. Data collection and analysis of manual work using video recording and personal computer techniques. International Journal of Industrial Ergonomics, 19 (4), 291-298.

Finnsgård, C., Wänström, C., Medbo, L. and Neumann, W.P., 2008. The impact of materials exposure on the conditions at the workstation. In: Proceedings of the 15th International Annual Euroma Conference. University of Groningen, the Netherlands, 15-18 June 2008.

Finnsgård, C. and Wänström, C., 2009. Factors impacting manual picking at assembly lines - an experiment in the automotive industry. In: Proceedings of the 16th International Annual Euroma Conference. Chalmers University of Technology, Gothenburg, Sweden, 14-17 June 2009.

Hanson, R., 2011. Effects of using minomi in in-plant materials supply. Journal of Manufacturing Technology Management, 22 (1).

Hua, S.Y. and Johnson, D.J., 2010. Research issues on factors influencing the choice of kitting versus line stocking. International Journal of Production Research, 48 (3), 779-800. 
Johansson, M.I., 1991. Kitting systems for small size parts in manual assembly systems. In: M. Pridham, and C. O'Brien, eds. Production research - approaching the 21 st century. London: Taylor \& Francis, 225-230.

Jonsson, D., Medbo, L. and Engström, T., 2004. Some considerations relating to the reintroduction of assembly lines in the Swedish automotive industry. International Journal of Operations \& Production Management, 24 (8), 754-772.

Medbo, L., 1999. Materials Supply and Product Descriptions for Assembly Systems. Thesis (PhD). Chalmers University of Technology, Göteborg.

Medbo, L., 2003. Assembly work execution and materials kit functionality in parallel flow assembly systems. International Journal of Industrial Ergonomics, 31, 263281.

Neumann, W.P., Winkel, J., Medbo, L., Magneberg, R., and Mathiassen, S.E., 2006. Production system design elements influencing productivity and ergonomics - A case study of parallel and serial flow strategies. International Journal of Operations \& Production Management, 26 (8), 904-923.

Sellers, C.J. and Nof, S.Y., 1986. Part kitting in robotic facilities. Material Flow, 3 (1-3), 163-174.

Tamaki, K. and Nof, S. Y., 1991. Design method of robot kitting system for flexible assembly. Robotics and Autonomous Systems, 8 (4), 255-273.

Wänström, C. and Medbo, L., 2009. The impact of materials feeding design on assembly process performance. Journal of Manufacturing Technology Management, 20 (1), 30-51.

Womack, J.P., Jones, D.T. and Roos, D., 1990. The machine that changed the world. New York: Rawson Associates.

Yin, R.K., 2003. Case study research - design and methods. Thousand Oaks, CA: Sage publications,. 
Table 1. Number of assembly cycles recorded and analysed in each case study.

\begin{tabular}{|l|l|c|}
\hline \multicolumn{2}{|c|}{} & Number of assembly cycles recorded \\
\hline \multirow{2}{*}{ Case study 1 } & Kitting & 9 \\
\cline { 2 - 3 } & No kitting & 5 \\
\hline \multirow{2}{*}{ Case study 2 } & Kitting & 13 \\
\cline { 2 - 3 } & No kitting & 10 \\
\hline \multirow{2}{*}{ Case study 3a } & Kitting & 2 \\
\cline { 2 - 3 } & No kitting & 2 \\
\hline \multirow{2}{*}{ Case study 3b } & Kitting & 2 \\
\cline { 2 - 3 } & No kitting & 9 \\
\hline
\end{tabular}


Table 2. Overview of the characteristics of case 1.

\begin{tabular}{|l|l|c|c|c|c|}
\hline \multicolumn{2}{|l|}{} & $\begin{array}{c}\text { Assembly } \\
\text { cycle time }\end{array}$ & $\begin{array}{l}\text { Distance between assembly } \\
\text { object and component racks }\end{array}$ & $\begin{array}{c}\text { Number of parts } \\
\text { presented in kits }\end{array}$ & $\begin{array}{c}\text { Number of parts presented } \\
\text { in component racks }\end{array}$ \\
\hline $\begin{array}{l}\text { Case } \\
\text { study } 1\end{array}$ & Kitting & $13 \mathrm{~min}$ & $\mathrm{n} / \mathrm{a}$ & All & 0 \\
\cline { 2 - 6 } & No kitting & $1.0 \mathrm{~min}^{\star}$ & $1.7 \mathrm{~m}$ & 0 & All \\
\hline
\end{tabular}

*1.0 min was the basic cycle time. Multiples of 2, 3 and 4 times this basic cycle time occurred. 
Table 3. Overview of the characteristics of case 2.

\begin{tabular}{|l|l|c|c|c|c|}
\hline \multicolumn{2}{|l|}{} & $\begin{array}{c}\text { Assembly } \\
\text { cycle time }\end{array}$ & $\begin{array}{c}\text { Distance between assembly } \\
\text { object and component racks }\end{array}$ & $\begin{array}{c}\text { Number of parts } \\
\text { presented in kits }\end{array}$ & $\begin{array}{c}\text { Number of parts presented } \\
\text { in component racks }\end{array}$ \\
\hline $\begin{array}{l}\text { Case } \\
\text { study 2 }\end{array}$ & Kitting & $2.0 \mathrm{~min}$ & $1.1 \mathrm{~m}$ & 3 & 3 \\
\cline { 2 - 6 } & No kitting & $2.7 \mathrm{~min}$ & $1.1 \mathrm{~m}$ & 0 & 9 \\
\hline
\end{tabular}


Table 4. Overview of the characteristics of case $3 \mathrm{a}$.

\begin{tabular}{|l|l|c|c|c|c|}
\hline \multicolumn{2}{|l|}{} & $\begin{array}{c}\text { Assembly } \\
\text { cycle time }\end{array}$ & $\begin{array}{c}\text { Distance between assembly } \\
\text { object and component racks }\end{array}$ & $\begin{array}{c}\text { Number of parts } \\
\text { presented in kits }\end{array}$ & $\begin{array}{c}\text { Number of parts presented } \\
\text { in component racks }\end{array}$ \\
\hline $\begin{array}{l}\text { Case } \\
\text { study 3a }\end{array}$ & Kitting & $1.6 \mathrm{~min}$ & $1.1 \mathrm{~m}$ & 2 & 7 \\
\cline { 2 - 6 } & No kitting & $2.1 \mathrm{~min}$ & $1.1 \mathrm{~m}$ & 0 & 10 \\
\hline
\end{tabular}


Table 5. Overview of the characteristics of case $3 b$.

\begin{tabular}{|l|l|c|c|c|c|}
\hline \multicolumn{2}{|l|}{} & $\begin{array}{c}\text { Assembly } \\
\text { cycle time }\end{array}$ & $\begin{array}{c}\text { Distance between assembly } \\
\text { object and component racks }\end{array}$ & $\begin{array}{c}\text { Number of parts } \\
\text { presented in kits }\end{array}$ & $\begin{array}{c}\text { Number of parts presented } \\
\text { in component racks }\end{array}$ \\
\hline $\begin{array}{l}\text { Case } \\
\text { study 3b }\end{array}$ & Kitting & $1.6 \mathrm{~min}$ & $1.1 \mathrm{~m}$ & 1 & 9 \\
\cline { 2 - 6 } & No kitting & $2.1 \mathrm{~min}$ & $1.1 \mathrm{~m}$ & 0 & 7 \\
\hline
\end{tabular}


Table 6. Overview of the findings of the four case studies - fetching times.

\begin{tabular}{|c|c|c|c|c|c|c|c|}
\hline & & $\begin{array}{c}\text { Proportion } \\
\text { of kitted } \\
\text { parts }\end{array}$ & $\begin{array}{l}\text { Average no. of parts } \\
\text { fetched from } \\
\text { component racks } \\
\text { each assembly cycle }\end{array}$ & \begin{tabular}{|c|} 
Average no. of \\
visits to component \\
racks each \\
assembly cycle \\
\end{tabular} & $\begin{array}{c}\text { Average } \\
\text { fetching time } \\
\text { per part - total }\end{array}$ & $\begin{array}{c}\text { Average } \\
\text { fetching time } \\
\text { per part from } \\
\text { kit }\end{array}$ & \begin{tabular}{|c|}
$\begin{array}{c}\text { Average fetching } \\
\text { time per part } \\
\text { from component } \\
\text { racks }\end{array}$ \\
\end{tabular} \\
\hline \multirow{2}{*}{$\begin{array}{l}\text { Case } \\
\text { study } 1\end{array}$} & Kitting & $100 \%$ & 0 & 0 & $2.81 \mathrm{~s}$ & $2.81 \mathrm{~s}$ & $\mathrm{n} / \mathrm{a}$ \\
\hline & No kitting & $0 \%$ & $3.3^{*}$ & $1.9^{*}$ & $7.55 \mathrm{~s}$ & $\mathrm{n} / \mathrm{a}$ & $7.55 \mathrm{~s}$ \\
\hline \multirow{2}{*}{$\begin{array}{l}\text { Case } \\
\text { study } 2\end{array}$} & Kitting & $50 \%$ & 3 & 1 & $1.86 \mathrm{~s}$ & $1.46 \mathrm{~s}$ & $2.26 \mathrm{~s}$ \\
\hline & No kitting & $0 \%$ & 9 & 7 & $3.29 \mathrm{~s}$ & $n / a$ & $3.29 \mathrm{~s}$ \\
\hline \multirow{2}{*}{$\begin{array}{l}\text { Case } \\
\text { study } \\
3 a\end{array}$} & Kitting & $22 \%$ & 7 & 3 & $3.14 \mathrm{~s}$ & $1.14 \mathrm{~s}$ & $3.71 \mathrm{~s}$ \\
\hline & No kitting & $0 \%$ & 10 & 3 & $3.07 \mathrm{~s}$ & $n / a$ & $3.07 \mathrm{~s}$ \\
\hline \multirow{2}{*}{$\begin{array}{l}\text { Case } \\
\text { study } \\
3 \text { b }\end{array}$} & Kitting & $10 \%$ & 9 & 4 & $2.19 \mathrm{~s}$ & $0.99 \mathrm{~s}$ & $2.32 \mathrm{~s}$ \\
\hline & No kitting & $0 \%$ & 7 & 4 & $2.35 \mathrm{~s}$ & $\mathrm{n} / \mathrm{a}$ & $2.35 \mathrm{~s}$ \\
\hline
\end{tabular}

* Average from 15 assembly stations with assembly cycles of 1, 2, 3 or 4 minutes 